\title{
A THEOREM ON THE RESTRICTION OF TYPE I REPRESENTATIONS OF A GROUP TO CERTAIN OF ITS SUBGROUPS ${ }^{1}$
}

\author{
ROBERT R. KALLMAN
}

Abstract. Theorem. Let $G$ be a separable locally compact group and $H$ a closed subgroup such that $G / H$ is finite. Let $\pi$ be a Type I representation of $G$. Then $\pi \mid H$ is Type I.

The purpose of this note is to prove the following theorem.

THEOREM 1. Let $G$ be a separable locally compact group and $H$ a closed subgroup such that $G / H$ is finite. Let $\pi$ be a Type I representation of $G$. Then $\pi / H$ is Type I.

The proof of this theorem is surprisingly intricate. Note that since $G$ is separable, we may assume that $\pi$ acts on a separable Hilbert space.

If $\boldsymbol{R}$ is a von Neumann algebra, let $\boldsymbol{P}(\boldsymbol{R})$ be the lattice of all projections in $\boldsymbol{R}$.

Lemma 2. Let $\boldsymbol{R}$ be an Abelian von Neumann algebra, $F$ a finite group and $a \rightarrow \varphi(a)$ a homomorphism of $F$ into the group of ${ }^{*}$-automorphisms of $\boldsymbol{R}$. Then there exists a sequence of nonzero, mutually orthogonal projections $\left[P_{n}\right]$ in $P(R)$ such that: (1) $\sum_{n=1} P_{n}=I$; (2) if $a \in F, Q \in P(R), Q \leqq P_{n}$ (for some $n$ ), then either $\varphi(a)(Q)=Q$ or $\varphi(a)(Q)$ is orthogonal to $P_{n}$.

Proof. Let $a \in F$. Recall (Kallman [2]) that there exists a projection $P(a, 1)$ in $P(R)$ such that if $Q \in P(R), Q \leqq P(a, 1)$, then $\varphi(a)(Q)=Q$, and if $Q \leqq I-P(a, 1)$, then there exists $Q^{\prime} \in P(R), 0 \neq Q^{\prime} \leqq Q$, such that $\varphi(a)\left(Q^{\prime}\right) \perp Q^{\prime}$. Hence, an easy application of Zorn's lemma shows that there exists a sequence of nonzero projections $[P(a, n) \mid n \geqq 2]$ such that $\varphi(a)(P(a, n)) \perp P(a, n)$ and $\sum_{n>2} P(a, n)=I-P(a, 1)$. Now consider the nonzero elements of $\left[\inf _{a \in F} P\left(a, i_{a}\right) \mid i_{a} \geqq 1\right]$. These are a countable set of projections in $P(\boldsymbol{R})$, whose sum is $I$, and a little thought shows that they satisfy (2). Q.E.D.

Received by the editors October 23, 1972.

AMS (MOS) subject classifications (1970). Primary 22D10, 46L10.

Key words and phrases. Operator theory, von Neumann algebras, group representations.

${ }^{1}$ Work performed under the auspices of the U.S. Atomic Energy Commission. 
LemMa 3. Let $\boldsymbol{R}$ be a von Neumann algebra with no finite Type I summands, $F$ a finite group, $a \rightarrow \varphi(a)$ a representation of $F$ into the group of ${ }^{*}$-automorphisms of $\boldsymbol{R}$ which leave Cent $\boldsymbol{R}$ pointwise fixed. Then there exists a noncentral operator $T$ in $\boldsymbol{R}$ which is fixed by every $\varphi(a)(a \in F)$.

Proof. Since $\boldsymbol{R}$ has no finite Type I summands, there exists a sequence $\left[P_{n} \mid n \geqq 1\right] \subset P(R)$ such that $P_{m} \perp P_{n}(m \neq n)$ and $C\left(P_{n}\right)=C\left(P_{n}\right)=C(m, n \geqq 1)$, where $C\left(P_{m}\right)$ is the central support of $P_{m}$. Consider $Q_{m}=\sum_{a \in F} \varphi(a)\left(P_{m}\right)$. If some $Q_{m}$ is noncentral, then we are done, for each $Q_{m}$ is clearly invariant under $F$. Hence, suppose $Q_{m}$ central for all $m \geqq 1$. Notice that if $C^{\prime} \in P($ Cent $R), C^{\prime} \leqq C$, then the support of $Q_{m} \cdot C^{\prime}$ is $C^{\prime}$. In particular, the support of $Q_{m}=C$. Also, $\left\|Q_{m} \cdot C^{\prime}\right\| \geqq\left\|P_{m} \cdot C^{\prime}\right\|=1$ since $P_{m} \cdot C^{\prime}$ is a nonzero projection in $\boldsymbol{R}$. Hence, $Q_{m} \geqq C$. But $Q_{m} \rightarrow 0$ in the strong operator topology. This is nonsense since $C$ is fixed and nonzero. Hence, some $Q_{m}$ is noncentral. Q.E.D.

LEMMA 4. For every integer $n \geqq 2$, there exists an $n \times n$ selfadjoint matrix with real entries which has zero diagonal, which has 1 as an eigenvalue with multiplicity 1 , and has $-1 /(n-1)$ as an eigenvalue with multiplicity $n-1$.

Proof. We merely write down such a matrix for every $n \geqq 2$. Let $T_{n}=\left(t_{i j}\right)$, where $t_{i i}=0(1 \leqq i \leqq n)$ and $t_{i j}=1 /(n-1)(i \neq j)$. Compute that $(1,1, \cdots, 1)$ is an eigenvector with eigenvalue 1 , and that $(-1,1$, $0, \cdots, 0),(0,-1,1,0, \cdots, 0), \cdots,(0, \cdots,-1,1)$ are eigenvectors with eigenvalue $-1 /(n-1)$.

Since these $n$ vectors are linearly independent, the lemma is proved. Q.E.D.

Lemma 5. Let $\boldsymbol{R}$ be a factor and $\boldsymbol{A} \subset \boldsymbol{R}$ an Abelian von Neumann subalgebra which has no minimal projections. Then for every integer $m \geqq 1$, there exists a Type $\mathrm{I}_{m}$ subfactor $\boldsymbol{S} \subset \boldsymbol{R}$ such that $\boldsymbol{A} \cap \boldsymbol{S}$ is maximal Abelian in $\boldsymbol{S}$.

Proof. First of all, note that for every $m \geqq 1$, there exist $m$ nonzero mutually orthogonal equivalent projections in $\boldsymbol{A}$ with sum $I$. This is clear if $\boldsymbol{R}$ is a Type III $_{\infty}$ factor, for choose any $m$ nonzero mutually orthogonal projections with sum $I$. Any two such projections are equivalent (remember our Hilbert space is separable) in a Type $\mathrm{III}_{\infty}$ factor.

Next, suppose $\boldsymbol{R}$ is a $\mathrm{I}_{\infty}$ or a $\mathrm{II}_{\infty}$ factor. First, suppose that there exist $Q_{1}, Q_{1}^{\prime} \in P(\boldsymbol{A}), Q_{1} \perp Q_{1}^{\prime}, Q_{1}$ and $Q_{1}^{\prime}$ infinite. If $Q_{2}=I-Q_{1}$, then $Q_{2} \in P(A)$, $Q_{2} \perp Q_{1}$, and $Q_{2}$ is infinite. Hence, $Q_{2}$ is equivalent to $Q_{1}$. If no such pair $Q_{1}, Q_{1}^{\prime}$ exists, then $I=\sum_{m \geqq 1} P_{m}$, where the $P_{m}$ are finite, mutually orthogonal projections in $P(A)$. Then an elementary exercise using the semifinite trace on $R$ shows that there exist subsets $Z_{1}, Z_{2} \subset Z^{+}$such that $Z^{+}=Z_{1} \cup Z_{2}$, $Z_{1} \cap Z_{2}=\varnothing, Q_{1}=\sum_{m \in Z_{1}} P_{m}$ is infinite, and $Q_{2}=\sum_{m \in Z_{2}} P_{m}$ is infinite. 
Hence, in either case, there exist $Q_{1}, Q_{2} \in P(A), Q_{1}$ and $Q_{2}$ infinite, $Q_{1}$ is equivalent to $Q_{2}$, and $Q_{1}+Q_{2}=I$. Consider $Q_{2} R Q_{2}$ and $Q_{2} A Q_{2} \cdot Q_{2} R Q_{2}$ is a $\mathrm{I}_{\infty}$ or $\mathrm{II}_{\infty}$ factor and $Q_{2} A Q_{2}$ is an Abelian von Neumann subalgebra with no minimal projections. Hence, as above, there exist two infinite orthogonal equivalent projections $Q_{2}^{\prime}$ and $Q_{3}^{\prime}$ in $Q_{2} A Q_{2}$ such that $Q_{2}=Q_{2}^{\prime}+Q_{3}^{\prime}$. But then $Q_{1}, Q_{2}^{\prime}, Q_{3}^{\prime}$ are three infinite orthogonal equivalent projections in $\boldsymbol{R}$ with sum $I$. Continuing this argument, one sees that for every $m \geqq 1$, there exist $m$ nonzero mutually orthogonal equivalent projections in $\boldsymbol{A}$ with sum $I$.

If $\boldsymbol{R}$ is a $\mathrm{II}_{1}$ factor, it suffices to show that there exists a projection $Q \in P(A)$ such that $\operatorname{trace}(Q)=1 / m$. There exists a nonzero projection $P \in P(A)$ such that $\operatorname{trace}(P)<1 / m$ since $A$ has no minimal projections. By Zorn's lemma choose a maximal chain $\left[Q_{\alpha}\right]$ of elements of $P(A)$, ordered by inclusion, such that $\operatorname{trace}\left(Q_{\alpha}\right) \leqq 1 / m$. Then $Q=\sup _{\alpha} Q_{\alpha} \in P(A)$. We claim that $\operatorname{trace}(Q)=1 / m$. If not, then $\operatorname{trace}(Q)<1 / m$. Then there exists a nonzero $Q^{\prime} \in P(A)$ such that $\operatorname{trace}\left(Q^{\prime}\right)<1 / m-\operatorname{trace}(Q)$ and $Q^{\prime} \leqq I-Q . Q^{\prime}$ exists since $\boldsymbol{A}$ has no minimal projections. But then $\left[Q_{\alpha}\right] \cup\left[Q+Q^{\prime}\right]$ is a properly larger chain than $\left[Q_{\alpha}\right]$, and $\operatorname{trace}\left(Q+Q^{\prime}\right) \leqq 1 / m$. This is a contradiction. Hence, $\operatorname{trace}(Q)=1 / m$.

$\boldsymbol{R}$ cannot be a finite Type I factor, for any Abelian von Neumann subalgebra of $\boldsymbol{R}$ is generated by its minimal projections.

Therefore, let $Q_{1}, \cdots, Q_{m}$ be $m$ nonzero orthogonal equivalent projections in $A$ with sum $I$. Let $W_{11}=Q_{1}$ and let $W_{1 j}(2 \leqq j \leqq m)$ be a partial isometry whose initial projection is $Q_{j}$ and whose final projection is $Q_{1}$. Set $W_{j 1}=W_{1 j}^{*}$ and $W_{i j}=W_{i 1} \cdot W_{1 j}$. Then $W_{i j}$ is a partial isometry with initial projection $Q_{j}$ and final projection. $Q_{i}, W_{i j} \cdot W_{k l}=\delta_{j k} \cdot W_{i l}$, and $W_{j j}=Q_{j}$. One checks easily that the linear span of the $W_{i j}(1 \leqq i, j \leqq m)$ is a selfadjoint algebra $S$ which is a Type $\mathrm{I}_{m}$ factor.

Finally, $\boldsymbol{S} \cap \boldsymbol{A} \supset$ the linear span of $Q_{1}, \cdots, Q_{m}$. But this linear span is a maximal Abelian selfadjoint subalgebra of $S$. Hence, $S \cap A$ actually equals this linear span. Q.E.D.

Lemma 6. Let $\boldsymbol{R}$ be a factor, $F$ a finite group, and $a \rightarrow \varphi(a)$ a homomorphism of $F$ into the group of *automorphisms of $\boldsymbol{R}$. Let $\boldsymbol{A}$ be the von Neumann subalgebra of $\boldsymbol{R}$ consisting of those elements of $\boldsymbol{R}$ which are left fixed by every $\varphi(a)(a \in F)$. Suppose $A$ is Abelian. Then $A$ is generated by its minimal projections.

Proof. It suffices to show that $\boldsymbol{A}$ has at least one nonzero minimal projection. Suppose not. Let $f$ be the number of elements in $F$. Choose a large integer $m$ such that $m-1>f$. By Lemma 5 , there exists an $m \times m$ matrix subalgebra $S$ of $R$ such that we may identify $A \cap S$ with the algebra of all diagonal matrices in $S$. Choose $0<\lambda_{1}<\lambda_{2}<\cdots<\lambda_{m}$ and let 
$A \in S \cap A$ be the matrix with $\lambda_{1}, \cdots, \lambda_{n}$ as diagonal elements. Let $T$ be the $m \times m$ matrix described in Lemma 4 . In a natural manner, $T \in S$. Define a third skew-adjoint matrix $M=\left(m_{i j}\right)$ in $S$ by $m_{i i}=0(1 \leqq i \leqq m)$ and $m_{i j}=$ $t_{i j} /\left(\lambda_{i}-\lambda_{j}\right)(i \neq j)$. One computes that $A \cdot M-M \cdot A=T$. For any $a \in F$, note that $A \cdot \varphi(a)(M)-\varphi(a)(M) \cdot A=\varphi(a)(T)$.

Since $A$ is the fixed point set of the $\varphi(a)(a \in F)$, we have that

$$
A \cdot \sum_{a \in F} \varphi(a)(M)=\sum_{a \in F} \varphi(a)(M) \cdot A, \quad \text { or } \sum_{a \in F} \varphi(a) T=0 .
$$

But this is not true. Let $T=T^{+}-T^{-}$be the decomposition of $T$ into its positive and negative parts. $\left\|T^{+}\right\|=1$ and $\left\|T^{-}\right\|=1 /(m-1)$ by Lemma 4 . Hence, $\left\|\sum_{a \in F^{*}} \varphi(a)\left(T^{+}\right)\right\| \geqq\left\|T^{+}\right\| \geqq 1$. But $\left\|\sum_{a \in F} \varphi(a)\left(T^{-}\right)\right\| \leqq f \cdot\left\|T^{-}\right\|<1$. Hence, $\sum_{a \in F} \varphi(a)(T)$ is nonzero. Hence, $\boldsymbol{A}$ must have at least one nonzero minimal projection. Q.E.D.

Let $\boldsymbol{Z}$ be an Abelian von Neumann algebra on the Hilbert space $\boldsymbol{K}$. Recall that there exists a standard Borel space $\Xi$, a Borel measure $\mu$ on $\Xi$, and a measurable field of separable Hilbert spaces $\xi \rightarrow K_{\xi}$ on $\Xi$ such that $\boldsymbol{K}=\int_{\Xi}^{\Theta} \boldsymbol{K}_{\xi} d \mu(\xi)$ and $\boldsymbol{Z}=L^{\infty}(\Xi, d \mu)$. Let $\boldsymbol{A}$ be a separable $C^{*}$-algebra and $a \rightarrow \pi(a)$ a *-representation of $\boldsymbol{A}$ on $\boldsymbol{K}$. We say that $\pi$ is nondegenerate if the identity operator $I$ is in the strong closure of $\pi(A)$.

Lemma 7. Let $\pi(A)$ commute with $Z$. Then for every $\xi \in \Xi$, there exists a ${ }^{*}$-representation $\pi_{\xi}(\cdot)$ of $\boldsymbol{A}$ on $\boldsymbol{K}_{\xi}$ such that $\xi \rightarrow \pi_{\xi}($ a) is measurable for every $a \in A$ and $\pi(a)=\int_{\Xi}^{\Theta} \pi_{\xi}(a) d \mu(\xi)$. There exists a $\mu$-null Borel set $N \subset \Xi$ such that for $\xi \in \Xi-N, \pi_{\xi}(\cdot)$ is nondegenerate.

Proof. The existence of the $\pi_{\xi}(\cdot)$ follows easily from Dixmier [1, Théorème 1, p. 167], since $\pi(A)$ has a countable dense subalgebra over the complex rationals. That $\pi_{\xi}(\cdot)$ is nondegenerate for $\mu$-almost all $\xi$ follows easily from Dixmier [1, Proposition 4, p.162], since $\pi(A)$ is nondegenerate. Q.E.D.

Henceforth, we can (and do) assume that $N=\varnothing$.

Proposition 8. Suppose that $\pi(\cdot)$ is Type I. Then there exists a $\mu$-null Borel set $N \subset \Xi$ such that for $\xi \in \Xi-N, \pi_{\xi}(\cdot)$ is Type $\mathrm{I}$.

Proof. Let $R$ be the von Neumann algebra generated by $Z$ and $\pi(A)$. $\boldsymbol{Z}$ and $\boldsymbol{R}(\pi)$ are both Type I and commute. Hence, $\boldsymbol{R}$ is Type I since any finite collection of commuting Type I von Neumann algebras generate a Type I von Neumann algebra. There exists a measurable field $\boldsymbol{\xi} \rightarrow \boldsymbol{R}(\boldsymbol{\xi})$ of von Neumann algebras on $\boldsymbol{K}_{\xi}$ such that $\boldsymbol{R}=\int_{\Xi}^{\Theta} \boldsymbol{R}(\xi) d \mu(\xi)$. Let

$$
Z_{1}=\int_{\Xi}^{\Theta} Z_{1}(\xi) d \mu(\xi), \quad Z_{2}=\int_{\Xi}^{\Theta} Z_{2}(\xi) d \mu(\xi), \cdots
$$


be a countable subset of $\boldsymbol{Z}$ which is strongly dense in $\boldsymbol{Z}$. We may assume that $Z_{n}(\xi)$ is a scalar for all $n \geqq 1$ and $\xi \in \Xi$. Since the $Z_{n}(n \geqq 1)$ and $\pi(A)$ generate $\boldsymbol{R}$, there exists a $\mu$-null Borel set $N_{1} \subseteq \Xi$ such that for $\xi \in \Xi-N_{1}$, $\boldsymbol{R}(\xi)$ is generated by $Z_{n}(\xi)(n \geqq 1)$ and $\pi_{\xi}(A)$ (see Dixmier [1, Théorème 1, p. 178]). Since $\boldsymbol{R}$ is Type I, there exists a $\mu$-null Borel set $N_{2}$ such that for $\xi \in \Xi-N_{2}, \boldsymbol{R}(\xi)$ is Type I (Dixmier [1, Proposition 7, p. 190]). Let $N=N_{1} \cup N_{2}$. Then for $\xi \in \Xi-N, \boldsymbol{R}(\xi)$ is Type I. Also $\pi_{\xi}(\boldsymbol{A})$ generates $\boldsymbol{R}(\xi)$ since each $Z_{n}(\xi)$ is a scalar and $\pi_{\xi}(\boldsymbol{A})$ is nondegenerate. Hence, for $\xi \in \Xi-N, \pi_{\xi}(\cdot)$ is Type I. Q.E.D.

PROOF OF THEOREM 1. Let $e=g_{1}, \cdots, g_{n}$ be coset representatives for $G / H . H^{\prime}=\bigcap_{1 \leqq i \leqq n} g_{i} \cdot H \cdot g_{i}^{-1}$ is normal in $G . G / H^{\prime}$ is finite by Poincaré's lemma. If we can show that $\pi \mid H^{\prime}$ is Type I, then $\pi \mid H$ is Type I by the main theorem of Kallman [3]. Hence, we may assume that $H$ is normal in $G$.

Consider $\boldsymbol{R}(\pi)$. Since $\pi$ is Type I, we may assume that $\boldsymbol{R}(\pi)^{\prime}$ is Abelian. Let us apply Lemma 2 to Cent $\boldsymbol{R}(\pi \mid H)$ and the finite group of *-automorphisms induced by $G / H$. Take some $P_{n} \cdot P_{n} \cdot R(\pi) \cdot P_{n}$ is Type $\mathrm{I}$ and is generated by $P_{n} \cdot \pi(g) \cdot P_{n}(g \in G)$. Now either $P_{n} \cdot \pi(g) \cdot P_{n}=0$ or $\pi(g)$ commutes with every projection $Q \in P_{n} \cdot$ Cent $R(\pi \mid H)$. Let $G^{\prime}=$ $\left[g \in G \mid P_{n} \cdot \pi(g) \cdot P_{n} \neq 0\right] . H \subset G^{\prime}, g \rightarrow P_{n} \cdot \pi(g)$ is a unitary representation of $G^{\prime}$, and the $P_{n} \cdot \pi(g)\left(g \in G^{\prime}\right)$ generate $P_{n} \cdot \boldsymbol{R}(\pi) \cdot P_{n}$. Now $\boldsymbol{R}(\pi \mid H)$ is Type I if and only if $P_{n} \cdot \boldsymbol{R}(\pi \mid H)$ is Type I for every $n \geqq 1$.

Hence, we may suppose that $H$ is normal in $G$, that $R(\pi)^{\prime}$ is Abelian, and that Cent $\boldsymbol{R}(\pi \mid H) \subset$ Cent $\boldsymbol{R}(\pi)$.

There exists a standard Borel space $\Xi$, a positive Borel measure $\mu$ on $\Xi$, a measurable field of separable Hilbert spaces $\xi \rightarrow K_{\xi}$, and a measurable field of strongly continuous unitary representations $\xi \rightarrow \pi_{\xi}$ of $G$ on $K_{\xi}$ such that $\boldsymbol{K}=\int_{\Xi}^{\Theta} \boldsymbol{K}_{\xi} d \mu(\xi), \pi(g)=\int_{\Xi}^{\Theta} \pi_{\xi}(g) d \mu(\xi)$, and Cent $\boldsymbol{R}(\pi \mid H)=L^{\infty}(\Xi, d \mu)$. Since Cent $\boldsymbol{R}(\pi \mid H) \subset$ Cent $\boldsymbol{R}(\pi)$, Proposition 8 implies that we may assume each $\pi_{\xi}$ is Type I. $\pi \mid H$ will be Type I if we can show that each $\pi_{\xi} \mid H$ is Type I since Cent $\boldsymbol{R}(\pi \mid H)=L^{\infty}(\Xi, d \mu)$. Hence, we may suppose $\pi \mid H$ is a factor.

$G / H$ has a natural homomorphism into the group of *-automorphisms of $\boldsymbol{R}(\pi \mid H)^{\prime}$ whose fixed point set is $\boldsymbol{R}(\pi)^{\prime}=$ Cent $\boldsymbol{R}(\pi)$. By Lemma 6, Cent $\boldsymbol{R}(\pi)$ is generated by its minimal projections.

Choose minimal orthogonal projections $Q_{1}, Q_{2}, \cdots \in P(\operatorname{Cent} \boldsymbol{R}(\pi))$ such that $\sum_{m \geq 1} Q_{m}=I . \boldsymbol{R}(\pi \mid H)$ is Type I if and only if $Q_{m} \cdot \boldsymbol{R}(\pi \mid H)$ is Type I for $m \geqq 1 . Q_{m} \cdot \boldsymbol{R}(\pi)$ is a Type I factor since $Q_{m}$ is a minimal projection in Cent $\boldsymbol{R}(\pi) \cdot Q_{m} \cdot \boldsymbol{R}(\pi \mid H)$ is also a factor. Hence, suppose that both $\boldsymbol{R}(\pi)$ and $\boldsymbol{R}(\pi \mid H)$ are factors and $\boldsymbol{R}(\pi)^{\prime}$ is the scalars.

Since $H$ is normal in $G, G / H$ has a natural homomorphism into the group of *-automorphisms of $\boldsymbol{R}(\pi \mid H)^{\prime}$. Since $\boldsymbol{R}(\pi)^{\prime}$ is the scalars, the fixed point set of $G / H$ acting on $R(\pi \mid H)^{\prime}$ is the scalars. Hence, Lemma 3 implies 
that $\boldsymbol{R}(\pi \mid H)^{\prime}$ is a finite Type I factor. Hence, $\boldsymbol{R}(\pi \mid H)$ is a Type I factor. Q.E.D.

\section{BIBLIOGRAPHY}

1. J. Dixmier, Les algèbres d'opérateurs dans l'espace hilbertien, Gauthier-Villars, Paris, 1957. MR 20 \#1234.

2. Robert R. Kallman, A generalization of free action, Duke Math. J. 36 (1969), 781-789. MR 41 \#838.

3. —_ Certain topological groups are type I, Bull. Amer. Math. Soc. 76 (1970), 404-406. MR 41 \#385.

Department of Mathematics, University of California, Los Alamos Scientific Laboratory, Los Alamos, New Mexico 87544

Current address: Department of Mathematics, University of Florida, Gainesville, Florida 32601 